01.X-03 REEIMEMENT OE TRNAEET :HOW TO COMBINE STEREOCHEMICAI INFORMATION WITH LOW AND MEDIUM RESOLUTION DATA. J.L. SUSSman, A.D. Podjarny, R.W. Schevitz and P. B. Sigler. Laboratorio de Rayos $X$, UNLP, Argentina; Department of Biophysics, U. of Chicago; Dept. Of Structural Chemistry, Weizmann Inst., Israel. Initiaton transfer RNA(tRNA Met) plays a central role in decoding the genetic information in messenger RNA (mRNA) during protein biosynthesis. In order to obtain the three-dimensional structure and to compare it with that of elongator tRNA, a crystallographic study waso undertaken. The resolution available was $4 \mathrm{~A}$. Data collection, heavy atom derivatives and direct method applications have already been described (Schevitz et al, (1979), Nature, 278 , 188-190; Podjarny et a1,(1981), in press, Acta Cryst. A). A model was obtained by interpreting the MIR electron density map with static and real time (Podjarny, Ph.D. thesis, 1976) computer graphics. This initial map was of very poor quality due to a consistent misassignment of the $z$-coordinate of the heavy atoms, which was verified after refinement of the entire structure. Such a systematic error was suspected since the R-factor was esentially random except at very low resolution. In order to correct it, a new model was obtained by fitting the molecular structure of tRNAPhe (Sussman et al, (1978), J.MOL.Biol., 123,607) to the computer graphics results for tRNA Met and refinement was started. This refinement proceeded in steps, initially with the smallest possible number of degrees of freedom (the entire structure treated as one rigid group) and only the lowest resolution data

$(12.5-20 \AA)$ in orden to maximize the radius of convergence. The number of degrees of freedom was then succesively increased to tro and four rigid groups corresponding to the different helical domains of the structure. This procedure requires previous knowledge of the stereochemistry and the secondary structure. The R-factor was lowered from $58 \%$ at $12.5 \AA$ to $33 \%$ at $12.5 \AA$, and then to $42 \%$ at $6 \AA$.The model was shifted by almost $5 \AA$, correcting the systematic error previously mentioned. Such a large displacement could only be obtained with the Iow resolution used at the first steps of the refinement. More data were subsequently used (first to $4.5 \AA$ and then to $4 \AA$ resolution) and the helical stereochemical constraints were relaxed. At this stage, the groups of known stereochemistry were separate phosphates and nucleosides, including WatsonCrick base pairing at the double-helical stem regions. Restraints were imposed between the groups to maintain reasonable stereochemistry and to prevent close contacts between non-bonded atoms.After 71 cycles of refinement at 4.5 $\AA$ and 21 cycles at $4 \AA$, the R-factor was $26.5 \%$. This procedure was monitored by examining difference electron density maps at virtually all stages; at one step where the refinement went into a false minimum, the model was corrected manually using a static graphics system. The final difference electron density map shows practically no features.

This whole procedure succeeded in correcting a large error with low resolution refinement and in providing a reasonable model by combining medium resolution data with a-priori

stereochemical knowledge.
01.X-04 RESTRAINED LEAST-SQUARES REFINEMENT: A USER'S PERSPECTIVE。 BY A.R. Sielecki, MRC Group in Protein Structure and Function, Department of Biochemistry, University of Alberta, Edmonton, Alberta, T6G 2H7, Canada.

Least-squares refinement of protein structures was pioneered by the Seattle group with rubredoxin (Watenpaugh, et a . Acta Cryst. B29:943 [1973]). There are now several other powerful refinement algorithms and we have used that of Hendrickson and Konnert (Biomolecular Structure, Function, Conformation \& Evolution, Ed. Srinivasan, Pergamon, Oxford 43 [1980]). The refinement experiences that will be described are based on the structures of three enzymes: SGPA and SGPB, which are bacterial serine proteases and penicillopepsin, a fungal aspartyl protease.

\section{SGPA SGPB penicillopepsin}

$\begin{array}{lcrr}\text { MoW. (daltons) } & 18,100 & 18,400 & 33,300 \\ \text { Res. (A) } & 12.0-1.5 & 8.0-1.7 & 8.0-1.8 \\ \text { HReflections } & 17,194 & 17,537 & 18,168 \\ \text { Present R factor } & 0.126 & 0.234 & 0.165\end{array}$

Each of these enzymes have required individual attention and the refinement pathways that were taken will be described. of particular importance for all three refinements has been the major contribution of resorting to $2 F_{0}-F_{c}$ and $F_{O}-F_{c}$ maps at key points during the refinement. Without exception each enzyme has had several residues in wrong conformations initially, which required adjustment before the refinement could proceed. Correction of these wrong conformations has been facilitated by the use of an MMS-X interactive graphics display. Software for this system has been developed by Colin Broughton, in our group.

The judicious choice of solvent molecules is also important to the progress of refinement. It would appear that some residues are strongly influenced by their close association with bound solvent and unless these atoms contribute to the structure factor calculation, false minima for residues of the protein molecule can result.

Emphasis will be placed on the advantages and drawbacks of the restrained parameter refinement program of Hendrickson. It is important to know the accuracy of the resulting coordinates. We have been able to obtain a measure of their precision by comparing the refined structures for SGPA (native) and for SGPA in complex with tetrapeptide product molecules and an inhibitor. These comparisons indicate that coordinates agree to within $0.06 \AA$ for the atoms of the main chain of SGPA in these several refinements.

(Research supported by the Medical Research Council of Canada.) 